\title{
Idade avançada e sexo são fatores de risco para falha na extubação em UTI adulto
}

\author{
Advanced age and gender are risk factors for extubation failure in adult ICU
}

\author{
(D) Marília Lambrecht da Silva, $\mathbf{M a}^{1}$ \\ (D) Anelise Sonza, $\mathbf{P h D}^{2}$ \\ (D) Dayane Montemezzo, $\mathbf{P h D}^{3}$ \\ Pedro Dal Lago, PhD $^{4}$
}

Parecer de Aprovação do Comitê de Ética em Pesquisa em Seres Humanos da Irmandade da Santa Casa de Misericórdia de Porto Alegre.

CAEE: 15048213.7 .0000 .5335

\section{Endereço para correspondência}

Prof. Dr. Pedro Dal Lago

Universidade Federal de Ciências da Saúde de Porto Alegre, Sarmento Leite, 245, CEP 90050-170, Porto Alegre, RS, Brasil.

Fax: +55 (51) 3303-8796

pdallago@ufcspa.edu.br

${ }^{1}$ Residência Multiprofissional Integrada em Saúde, Universidade Federal de Ciências da Saúde de Porto Alegre e Irmandade da Santa Casa de Misericórdia de Porto Alegre, Porto Alegre, RS, Brasil; Programa de Pós-graduação em Ciências da Reabilitação, Universidade Federal de Ciências da Saúde de Porto Alegre, Porto Alegre, RS, Brasil.

marilia_lambrecht@yahoo.com.br

\footnotetext{
${ }^{2}$ Departamento de Fisioterapia, Programa de Pós-graduação em Fisioterapia da Universidade do Estado de Santa Catarina, Florianópolis, SC, Brasil. Programa de Pós-graduação em Ciências do Movimento Humano da Universidade do Estado de Santa Catarina, Florianópolis, SC, Brasil.

anelise.sonza@udesc.br
}

\footnotetext{
${ }^{3}$ Departamento de Fisioterapia, Programa de Pós-graduação em Fisioterapia da Universidade do Estado de Santa Catarina, Florianópolis, SC, Brasil.

dayane.montemezzo@udesc.br

${ }^{4}$ Programa de Pós-graduação em Ciências da Reabilitação, Universidade Federal de Ciências da Saúde de Porto Alegre, Porto Alegre, RS, Brasil. Residência Multiprofissional Integrada em Saúde, Universidade Federal de Ciências da Saúde de Porto Alegre e Irmandade da Santa Casa de Misericórdia de Porto Alegre, Porto Alegre, RS, Brasil.

pdallago@ufcspa.edu.br
}

\begin{abstract}
Resumo
Introdução: A falha no processo de retirada da ventilação mecânica invasiva (VMI) está associada a desfechos adversos, como alta mortalidade hospitalar e custos elevados.

Objetivo: Conhecer os fatores de risco para falha na extubação em adultos internados em UTI submetidos à VMI. Secundariamente, conhecer a taxa de reintubação orotraqueal (Re-TOT).

Métodos: Estudo prospectivo, observacional, realizado em prontuários eletrônicos em um período de 11 meses em uma UTI adulto. Os testes Quiquadrado ou Exato de Fischer compararam a Re-TOT, sexo e faixas etárias. Resultados: Prontuários de 224 adultos foram analisados, com média de idade $61,9 \pm 16,9$ anos e $48,2 \%$ do sexo masculino. O principal motivo de internação foi por insuficiência respiratória aguda (33\%). Mulheres foram susceptíveis à falha na extubação comparadas aos homens $(21,5 \%$ vs. $10 \%$, $p=0,019$; respectivamente), e idade avançada. A Re-TOT foi de $31,5 \%$. Conclusão: Sexo feminino e idade avançada foram fatores de risco para falha na extubação. A Re-TOT foi mais elevada que outros índices da literatura.
\end{abstract}

Palavras-chave: Unidades de Terapia Intensiva. Extubação. Ventilação Mecânica. Desmame.

\begin{abstract}
Introduction: Failure in the invasive mechanical ventilation (IMV) withdrawal process is associated with adverse outcomes such as high hospital mortality and high costs.

Objective: To know the risk factors for extubation failure in an adult intensive care unit (ICU) undergoing IMV. Secondly, to know the rate of orotracheal reintubation (Re-TOT).

Methods: Prospective, observational study conducted on electronic medical records over a period of 11 consecutive months in an adult ICU. Chi-square or Fischer's Exact tests compared Re-TOT, sex and age groups.

Results: Medical records from 224 adults were analyzed, with a mean age of $61.9 \pm 16.9$ years and $48.2 \%$ male. The main reason for hospitalization was due to acute respiratory failure (33\%). Women were susceptible to extubation failure compared to men $(21.5 \%$ vs. $10 \%, p=0.019$; respectively), and advanced age. Re-TOT was $31.5 \%$.

Conclusion: Female gender and advanced age were risk factors for extubation failure. Re-TOT was higher than other indexes in the literature.
\end{abstract}

Keywords: Intensive Care Unit. Airway Extubation. Respiration. Artificial. Weaning.

\section{Cite como \\ Vancouver}

Silva, MLS, Sonza, A, Montemezzo, D, Lago, PLD. Idade avançada e sexo são fatores de risco para falha na extubação em UTI adulto. Conscientiae Saúde 2020;19(1):1-12, e16415. https://doi.org/10.5585/conssaude.v19n1.16415. 


\section{Introdução}

A ventilação mecânica invasiva (VMI) é utilizada convencionalmente em pacientes com doença respiratórias de diferentes etiologias ${ }^{1,2}$ e trata-se de um método de suporte respiratório ao paciente. A VMI pode ser utilizada preventivamente e para salvar vidas ${ }^{3-5}$, porém, não é considerada uma terapia curativa. Devido a sua associação com complicações, pacientes devem ser liberados do ventilador o mais rápido para que possam sustentar uma respiração espontânea não assistida ${ }^{5}$. Modalidades ventilatórias e ventiladores foram desenvolvidos, possibilitando terapias menos agressivas ao paciente e, ao mesmo tempo, mais eficientes. A VMI continua a ser o principal tratamento médico para insuficiência respiratória aguda, e grande parte dos pacientes em unidades de terapia intensiva (UTI) necessita de ventilação mecânica ${ }^{4}$.

A cada ano, milhões de pacientes são admitidos nas UTI em todo o mundo e, em função dos avanços no manejo dos pacientes críticos e nas modalidades ventilatórias, muitos têm sobrevivido $^{6}$ e o tempo de permanência em VMI tem se tornado cada vez maior. Nos Estados Unidos, os pacientes sob VMI consomem 13,4\% de todos os recursos utilizados em um hospital (6). Além disso, o número de pacientes que necessitam de VMI prolongada aumenta 5,1\% ao ano, superando o crescimento anual da taxa de internação, que é de $1,2 \%{ }^{7}$. Ainda nos EUA, 66\% dos adultos com idade entre 71-80 anos ventilados mecanicamente vieram a óbito antes da alta hospitalar e 44\% daqueles entre 51-60 anos e uma mediana de custos hospitalares de 27 a 39 mil dólares por internação ${ }^{6}$.

O tempo prolongado de VMI pode induzir a diversas complicações ao paciente, aumentando o risco de morbi-mortalidades, sendo considerado um problema de saúde pública $^{1,5,6}$. Por esta razão, a partir da resolução da doença de base, tem-se início o processo de descontinuação da VMI, com adoção de estratégias para promover um oportuno e bemsucedido desmame da ventilação mecânica, o que deve ser considerado uma prioridade, a fim de melhorar a qualidade deste processo ${ }^{8,9}$.

O processo de desmame da VMI constitui um desafio e despende atenção da equipe intensivista, sendo, ainda, um dos problemas mais difíceis na prática clínica ${ }^{9}$, já que muitos dos pacientes internados em UTI apresentam falha na extubação (retorno para VMI nas primeiras 48 horas após a retirada da prótese endolaríngea $)^{10}$. A falha no processo de retirada da VMI está associada a desfechos adversos, incluindo alta mortalidade hospitalar, maior tempo de hospitalização e custos mais elevados, além de aumento nas taxas de reintubação. Embora ainda não se saiba qual é a taxa aceitável de reintubação, há relatos de que a taxa varia de 2 a $25 \%$ entre pacientes com extubação planejada ${ }^{2}$. Além disto, pacientes com falha de extubação 
apresentam pior prognóstico e aumento na taxa de traqueostomia ${ }^{11}$. O tempo total de exposição à VMI é significativamente associado com o aumento da mortalidade UTI, o que aumenta em até 9 dias para pacientes submetidos à reintubação ${ }^{12}$.

A taxa de mortalidade resultante de falha na extubação pode chegar a $50 \%$, o que indica o potencial de gravidade desse tipo de evento, e a necessidade de reintubação após falha na extubação está associada ao aumento do tempo de permanência na UTI ${ }^{11}$. Assim, é importante a identificação precoce de pacientes com necessidade de reintubação e a consequente reinstituição do suporte ventilatório, a fim de evitar que sejam readmitidos em UTI, o que está relacionado a um maior consumo de recursos financeiros e maior mortalidade hospitalar.

Frente ao exposto, o objetivo deste estudo foi conhecer os fatores de risco para falha na extubação em adultos internados em terapia intensiva submetidos à VMI. Secundariamente, conhecer a taxa de reintubação orotraqueal.

\section{Método}

Trata-se de um estudo prospectivo realizado na UTI geral de um hospital do Complexo Hospitalar Santa Casa de Misericórdia de Porto Alegre-RS. A UTI estudada, no período de realização deste estudo, admitia pacientes com idade superior a 18 anos, em situação crítica ou semicrítica, com 24 leitos, sendo cinco destes leitos destinados a pacientes que necessitavam de algum tipo de isolamento. Os dados foram coletados em prontuários eletrônicos dos pacientes no período de outubro de 2012 a agosto de 2013, e transferidos para uma planilha eletrônica do Microsoft Excel (versão 2011). O estudo foi aprovado pelo Comitê de Ética em Pesquisa da instituição sob o Protocolo 619.022/2013.

Foram elegíveis pacientes internados na UTI durante um período de 11 meses consecutivos, de ambos os sexos, com idade igual e/ou superior a 18 anos, sendo incluídos aqueles submetidos à VMI. Todos os pacientes que realizaram procedimento de traqueostomia antes da primeira extubação foram excluídos, conforme fluxograma do estudo representado na Figura 1. 
Figura 1 - Fluxograma de coleta de dados de pacientes internados na Unidade de Terapia Intensiva

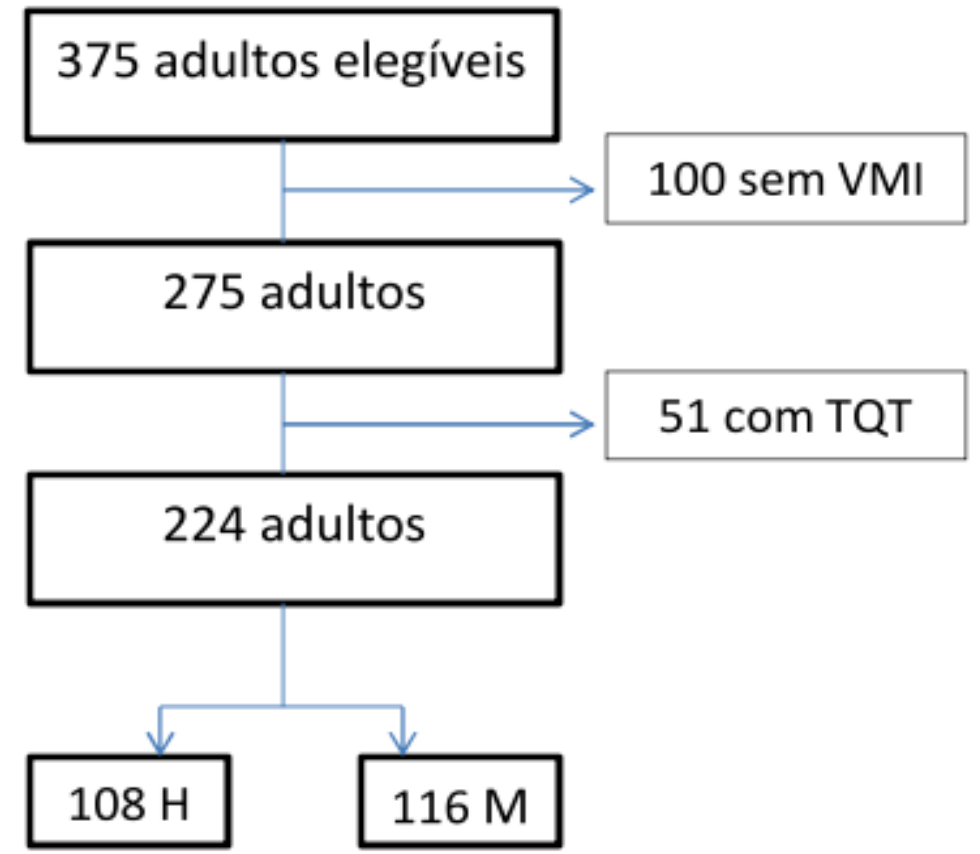

Legenda: VMI, ventilação mecânica invasiva; TQT, traqueostomia; H, homem; M, mulher. Fonte: Dados dos autores.

Para a análise dos dados, foi realizada a codificação de cada uma das variáveis do estudo, por meio de banco de dados em programa Microsoft Excel (versão 2011). Após, os dados foram transferidos para o programa Statistical Package for the Social Science (SPSS), versão 19.0. A distribuição normal dos dados foi analisada por meio do teste de Kolmogorov Smirnov. Os dados foram apresentados como medidas de tendência central, dispersão e frequência, absoluta (n) e/ou relativa (\%). Os testes Qui-quadrado ou teste exato de Fischer foram aplicados para analisar a associação entre taxa Re-TOT e sexo, e faixas etárias. Para significância estatística, foi considerado $\mathrm{p}<0,05$.

\section{Resultados}

Os dados demográficos e principais condições de saúde dos 224 indivíduos incluídos neste estudo, no momento da internação, por meio de prontuário eletrônico, são apresentados na Tabela 1. Em torno de $52 \%$ da amostra foi do sexo feminino e a variação de idade do grupo como um todo foi de 19 a 94 anos. Com relação ao motivo de internação, 33,1\% dos pacientes foram internados na UTI por insuficiência respiratória aguda (IRA) e outras doenças pulmonares, seguido de cirurgias $(27,2 \%)$, sepse $(10,3 \%)$ e rebaixamento do nível de consciência $(9,4 \%)$, sendo considerados os principais motivos de internação. Outros motivos 
como infarto agudo do miocárdio $(7,1 \%)$, acidente vascular encefálico $(4,5 \%)$ e outras causas, como encefalopatia, diabetes, aneurisma, neoplasia e convulsões fazem parte das demais causas das internações.

Tabela 1 - Dados demográficos e principais condições de saúde associadas da amostra no momento da internação $(\mathrm{n}=224)$, divididos por gênero

\begin{tabular}{lccc}
\hline & Feminino & Masculino & Total \\
\hline Gênero (n/ \%) & $116 / 51,8 \%$ & $108 / 48,2 \%$ & $224 / 100 \%$ \\
Idade (Média \pm DP) & $64,1 \pm 17$ & $59,6 \pm 6,4$ & $61,9 \pm 16,9$ \\
Condições de saúde (n/ \%) & & & \\
Insuficiência Respiratória Aguda & $28 / 12,5 \%$ & $27 / 12,1 \%$ & $55 / 24,6 \%$ \\
Broncopneumonia e broncoespasmo & $4 / 1,8 \%$ & $8 / 3,6 \%$ & $12 / 5,4 \%$ \\
Acidente Vascular Encefálico & $4 / 1,8 \%$ & $6 / 2,7 \%$ & $10 / 4,5 \%$ \\
Infarto Agudo do Miocárdio & $9 / 4 \%$ & $7 / 3,1 \%$ & $16 / 7,1 \%$ \\
Encefalopatia & $0 / 0 \%$ & $3 / 1,3 \%$ & $3 / 1,3 \%$ \\
Edema agudo de Pulmão & $3 / 1,3 \%$ & $2 / 0,9 \%$ & $5 / 2,2 \%$ \\
Sepse & $7 / 3,1 \%$ & $16 / 7,1 \%$ & $23 / 10,2 \%$ \\
Rebaixamento do Sensório & $13 / 5,8 \%$ & $8 / 3,6 \%$ & $21 / 9,4 \%$ \\
Outros (Diabetes, Neoplasia, DPOC, & $3 / 1,3 \%$ & $5 / 2,2 \%$ & $8 / 3,5 \%$ \\
Convulsões, Aneurisma) & & & $71 / 31,7 \%$ \\
Não especificado & $45 / 20,1 \%$ & $26 / 11,6 \%$ & \\
\hline
\end{tabular}

Fonte: Dados dos autores.

$\mathrm{Na}$ Tabela 2, pode-se observar que 94 pacientes foram a óbito antes de serem retirados da VMI, restando 130 pacientes elegíveis para ser verificada a taxa de reintubação orotraqueal na UTI. Outros 20 pacientes foram a óbito em período após a reintubação orotraqueal, totalizando 114 óbitos (50,9\%). A falha de extubação, bem como a reintubação orotraqueal, ocorreu em 41 pacientes $(31,5 \%)$. O gênero feminino foi considerado fator de risco para reintubação ( $p=0,019)$, quando comparado aos homens que também foram extubados. 
Tabela 2 - Êxitos e falhas na extubação e motivos de internação dos pacientes na UTI

\begin{tabular}{llll}
\hline & Feminino & Masculino & Total \\
\hline VMI & $116(51,8 \%)$ & $108(48,2 \%)$ & $224(100 \%)$ \\
Óbitos & $44(19,6 \%)$ & $50(22,3 \%)$ & $94(41,9 \%)$ \\
Êxito na extubação & $44(19,6 \%)$ & $45(20,2 \%)$ & $89(39,8 \%)$ \\
Re-TOT & $28(12,5 \%) *$ & $13(5,8 \%)$ & $41(18,3 \%)$
\end{tabular}

Legenda: VMI: ventilação mecânica invasiva; Re-TOT: reintubação orotraqueal; IRA: insuficiência respiratória aguda; IAM: infarto agudo do miocárdio. * Teste qui-quadrado comparando taxa Re-TOT entre sexos $(\mathrm{p}<0,05)$. Fonte: Dados dos autores.

Os principais motivos de reintubação e óbitos, por faixa etária e gênero, estão descritos na Tabela 3, tendo a depressão do sensório o maior percentual (39\%). Aproximadamente, 70\% dos motivos de reintubação são explicados pela depressão do sensório, fadiga, aumento da secreção e piora ventilatória. O tempo médio de VMI, em dias, dos pacientes que tiveram falha de extubação foi de $5,93 \pm 4,1$ dias.

Não foi encontrada diferença estatisticamente significativa na associação entre falha de extubação e motivo de internação na UTI $(p=0,968)$.

Ao compararmos a taxa de reintubação e as diferentes faixas etárias dos pacientes avaliados, foi encontrada diferença estatística entre as diferentes faixas etárias $(p=0,005)$. Entre 60 e 79 anos, foram encontrados os maiores índices de falha na extubação com subsequente reintubação $(56 \%)$.

Tabela 3 - Óbitos, taxa de reintubação (Re-TOT) e motivos de Re-TOT por faixa etária e gênero

\begin{tabular}{llllll}
\hline Idade & Óbitos & \multicolumn{3}{l}{ Re-TOT } & Motivos de Re-TOT \\
(anos) & Óbitos/ total & \multicolumn{3}{l}{ Re-TOT/ total } \\
\hline & $\mathrm{M}$ & $\mathrm{F}$ & $\mathrm{M}$ & $\mathrm{F}$ & Motivo $^{\ddagger}$ frequência (\% por faixa etária) \\
$18-39^{\S}$ & $8 / 17$ & $3 / 11$ & $1 / 9$ & $0 / 8$ & $1(100 \%)$ \\
$40-59^{*}$ & $15 / 27$ & $10 / 27$ & $2 / 12$ & $6 / 17$ & $1(37,5 \%), 2(37,5 \%), 4(12,5 \%), 5(12,5 \%)$ \\
$60-79^{*}$ & $31 / 546 / 1029 / 58$ & $7 / 23$ & $16 / 29$ & $1(34,8 \%), 2(17,5 \%), 3(13 \%) 4(21,7 \%), 5(13 \%)$ \\
$80-{ }^{*}$ & $60 / 108$ & $12 / 20$ & $3 / 4$ & $6 / 8$ & $1(44,4 \%), 2(11,1 \%), 3(11,1 \%) 4(22,3 \%), 5(11,1 \%)$ \\
Total & & $54 / 116$ & $13 / 48$ & $28 / 62$ &
\end{tabular}

Legenda: Re-TOT: reintubação orotraqueal; M, masculino; F, feminino; ${ }^{\ddagger}$ Motivos: 1 (rebaixamento do nível de consciência); 2 (fadiga); 3 (acumulo de secreção); 4 (piora ventilatória); 5 (outros). * Teste qui quadrado e $\$$ Teste exato de Fischer comparando taxa Re-TOT e faixas etárias $(\mathrm{p}<0,05)$.

Fonte: Dados dos autores. 


\section{Discussão}

Um dos objetivos das UTI é reduzir a mortalidade pela provisão de cuidados e observação individualizada contínua e integral, de acordo com a necessidade do paciente. Assim, conhecer os fatores de risco e a taxa de reintubação orotraqueal em adultos submetidos à VMI em uma UTI foram objetivos deste estudo. Muitos destes pacientes se encontram em situação crítica e na dependência da VMI, a grande maioria dos pacientes internados em UTI pertence à população com idade acima de 60 anos. Nesse estudo, a média de idade foi de 61,91 anos e 63,4\% dos pacientes submetidos à VMI tinham idade superior a 60 anos. A idade avançada representa risco para aumento na taxa de falha de extubação ${ }^{6}$, o que corrobora com os achados do presente estudo.

A maioria dos pacientes que recebem VMI apresenta insuficiência respiratória aguda, pneumonia, tromboembolismo, sepse, síndrome do desconforto respiratório agudo, atelectasias $^{1}$, além de causas como rebaixamento do nível de consciência e condição cirúrgica, adicionalmente observados em nosso estudo. Outros trabalhos corroboram com nossos resultados, porém com diferentes porcentagens para diferentes causas. No estudo de Danaga et al., dos 73 pacientes, 51 foram admitidos na UTI por motivos cirúrgico (70\%), enquanto que $22(30 \%)$ devido a diagnóstico clínico ${ }^{13}$. Outros autores identificaram a insuficiência respiratória como indicação mais frequente para admissão em UTI (47\%)(14). Apesar de algumas semelhanças entre os motivos de internação, há uma grande heterogeneidade entre os pacientes internados em UTI, bem como existem unidades diferenciadas, especializadas em dar suporte a determinados tipos de condições de saúde.

Em geral, pacientes que necessitam de tratamento intensivo apresentam quadro de saúde grave e esta condição está relacionada ao aumento nas taxas de mortalidade, inclusive a falha de extubação está associada com alta taxa de mortalidade ${ }^{2}$. No estudo de Louriz et al., sobre a decisão de negar ou retardar a internação na UTI, a mortalidade hospitalar foi de 33,3\% para os pacientes internados imediatamente na UTI, 43,8\% para pacientes internados tardiamente e 49,3\% para pacientes nunca admitidos em UTI(15). Neste estudo, 50,9\% (n = 114) dos pacientes internados foram a óbito, uma taxa superior àquelas citadas nos estudos acima reportados ${ }^{15}$, entretanto, vale ressaltar que grande parte dos pacientes do estudo possuía idade avançada. No entanto, isto pode ser em partes justificado pela gravidade do quadro dos pacientes e pelo fato de pacientes que ainda necessitam de tratamento intensivo ter sua alta antecipada devido à necessidade de liberação de leitos para a internação de pacientes mais graves, uma realidade do sistema de saúde no Brasil. 
Ainda em UTI, existe uma parcela de pacientes que não completa o tempo programado de desmame e precisa retornar para a VMI, além de um número de pacientes que, após serem extubados, precisa ser reintubado. Este processo está relacionado ao maior risco de infecção, tempo de VMI prolongada e custos hospitalares adicionais, o que leva a um aumento na taxa de mortalidade destes pacientes ${ }^{16}$. A diversidade entre os pacientes e diferentes intervenções clínicas impedem uma definição quanto à taxa de falha de extubação em UTI, no entanto, a literatura tem relatado que a taxa varia de 2 a $25 \%$ para pacientes com extubação planejada e $60 \%$ para extubações não planejadas ${ }^{2}$. Oliveira et al. relatam que $20 \%$ dos pacientes necessitam de reintubação logo após o processo de extubação, ou seja, entre os 88 pacientes que haviam passado no teste de respiração espontânea, 15 (17\%) pacientes necessitaram de reintubação em até 48 horas após a extubação ${ }^{17}$. Para nosso estudo, a taxa de falha de extubação, bem como a taxa de reintubação orotraqueal, foi de $31,5 \%(n=41)$, proporção que se encontra superior quando comparada a outros estudos.

Outras pesquisas também revelaram uma taxa de falha de extubação menor que a encontrada nesse trabalho e com valores de acordo com o previsto na literatura. Uma coorte de 119 pacientes com traumatismo cranioencefálico obteve sucesso na extubação em 104 pacientes $(87,4 \%)$ e $15(12,6 \%)$ foram reintubados ${ }^{18}$. Já Menon et al., após analisarem 2007 pacientes críticos, observaram que, em geral, 376 pacientes (19\%) necessitaram de, pelo menos, uma reintubação durante a internação ${ }^{19}$. Em outro estudo, clínico prospectivo, no qual todos os pacientes foram extubados após 30 minutos de teste de respiração espontânea bem-sucedida, também foi encontrada taxa de reintubação semelhante ao previsto na literatura, que foi de $22,8 \%{ }^{20}$. De acordo com Gao et al., atenção particular deve ser dada a pacientes após cirurgia cardíaca e àqueles que experienciaram uma extubação não planejada durante VMI, com aumento dos riscos de falha na extubação e mortalidade ${ }^{2}$.

A partir da falha de extubação, torna-se necessário o conhecimento dos fatores de risco e das ferramentas preditivas da falência da extubação ${ }^{2}$. Reis et al. apontam como causas principais de reintubação a insuficiência respiratória, a alteração no nível de consciência e o excesso de secreção pulmonar ${ }^{18}$, o que corrobora com os achados do nosso estudo, o qual aponta que, aproximadamente, $60 \%$ dos pacientes tiveram como causas de reintubação rebaixamento do nível de consciência e piora ventilatória. Além destes fatores, a extubação não planejada, tempo de VMI prolongado, cirurgia cardíaca, desordens de vias aéreas superiores, pneumonia e falhas em tentativas de desmame anteriores também estão relacionadas com aumento na taxa de reintubação orotraqueal ${ }^{2}$.

Outro aspecto relacionado à falha de extubação que merece ser destacado é sua possível 
relação com o sexo, assim como a faixa etária que podem ser fatores determinantes para o insucesso no desmame da $\mathrm{VMI}^{6}$. Neste estudo, houve maior prevalência de falha na extubação de pacientes do sexo feminino e, de forma geral, na faixa etária entre 60 e 79 anos. Em outro estudo, o grupo de sucesso da extubação apresentou maior proporção de homens $(96,2 \%$ versus $73,3 \% ; p=0,009)^{18}$, neste estudo, o maior percentual de insucesso foi, também, observado no sexo feminino, com $68,3 \%$ versus $31,7 \% ; p=0,019$ ). Em adição, os avanços na terapia intensiva têm favorecido um aumento na sobrevida de pacientes críticos/crônicos, aliados a inúmeros esforços que vêm sendo empregados na tentativa de reduzir o tempo de VMI e de estadia nas $\mathrm{UTI}^{5}$. Com isto, faz-se necessário saber identificar a presença de sinais e sintomas de desconforto respiratório nas primeiras 48 horas após a extubação a fim de conhecer as razões dadas para a sua falha, que incluem falta de melhoria no trabalho respiratório, hipoxemia, acidose respiratória, retenção de secreção e diminuição da consciência $(5,21)$ a fim de reduzir as taxas de reintubação orotraqueal e as complicações relacionadas a tal.

A ventilação mecânica é um processo invasivo, caro e, por si só, está associada com complicações potencialmente graves, juntamente com a falha na extubação, há um aumento dos desfechos desfavoráveis, incluindo altas taxas de mortalidade. No entanto, algumas estratégias têm demonstrado ser eficazes na promoção do desmame, e incluem protocolos de desmame e o uso de tentativas de respiração espontânea, além de monitoramento do delírio e mobilização precoce $^{8}$, sendo o teste de respiração espontânea (TRE) usualmente defendido como o melhor método para determinar a extubação dos pacientes ${ }^{22}$.

Tendo em vista que a taxa de reintubação orotraqueal nesta UTI foi superior ao previsto na literatura, espera-se que estes resultados sirvam como referência epidemiológica para rever os critérios de desmame/extubação da VMI, bem como, a utilização de protocolos padronizados, a fim de garantir requisitos de eficiência e segurança deste procedimento. Cabe ressaltar a importância deste estudo para a identificação e caracterização da população admitida na UTI.

Como limitações deste estudo, primeiramente, devido à falta de padronização nas avaliações das equipes de saúde, nem todas as informações requeridas estavam registradas no sistema eletrônico. Segundo, trata-se de um estudo observacional com dados geradores de hipótese, realizado em um centro único, o que dificulta a generalização dos resultados.

\section{Conclusão}

O sexo feminino e a idade acima de 60 anos foram fatores de risco para falha na 
extubação na amostra estudada. O registro da taxa de reintubação orotraqueal nos casos estudados desta UTI foi mais elevado que os índices apontados na literatura.

\section{Referências}

1. Klompas M, Anderson D, Trick W, Babcock H, Kerlin MP, Li L, et al. The preventability of ventilator-associated events: The CDC Prevention Epicenters Wake Up and Breathe Collaborative. Am J Respir Crit Care Med. 2015;191(3):292-301.

2. Gao F, Yang LH, He HR, Ma XC, Lu J, Zhai YJ, et al. The effect of reintubation on ventilator-associated pneumonia and mortality among mechanically ventilated patients with intubation: A systematic review and meta-analysis. Hear Lung J Acute Crit Care [Internet]. 2016;45(4):363-71. Available from: http://dx.doi.org/10.1016/j.hrtlng.2016.04.006

3. Perkins GD, Mistry D, Gates S, Gao F, Snelson C, Hart N, et al. Effect of Protocolized Weaning with Early Extubation to Noninvasive Ventilation vs Invasive Weaning on Time to Liberation from Mechanical Ventilation among Patients with Respiratory Failure: The Breathe Randomized Clinical Trial. JAMA - J Am Med Assoc. 2018;320(18):1881-8.

4. Amado-Rodríguez L, del Busto C, García-Prieto E, Albaiceta GM. Mechanical ventilation in acute respiratory distress syndrome: The open lung revisited. Med Intensiva [Internet]. 2017;41(9):550-8. Available from: http://dx.doi.org/10.1016/j.medin.2016.12.012

5. Girard TD, Alhazzani W, Kress JP, Ouellette DR, Schmidt GA, Truwit JD, et al. An Official American Thoracic Society/American College of Chest Physicians Clinical Practice Guideline: Liberation from mechanical ventilation in critically ill adults rehabilitation protocols, ventilator liberation protocols, and cuff leak tests. Am J Respir Crit Care Med. 2017;195(1):120-33.

6. Hayman WR, Leuthner SR, Laventhal NT, Brousseau DC, Lagatta JM. Cost comparison of mechanically ventilated patients across the age span. J Perinatol. 2015;35(12):1020-6.

7. Zilberberg MD, de Wit M, Shorr AF. Accuracy of previous estimates for adult prolonged acute mechanical ventilation volume in 2020. Crit Care Med [Internet]. 2012 Jan;40(1):1820. Available from: https://insights.ovid.com/crossref?an=00003246-201201000-00004

8. Rose L. Strategies for weaning from mechanical ventilation: A state of the art review. Intensive Crit Care Nurs [Internet]. 2015 Aug;31(4):189-95. Available from: https://linkinghub.elsevier.com/retrieve/pii/S0964339715000464

9. Pu L, Zhu B, Jiang L, Du B, Zhu X, Li A, et al. Weaning critically ill patients from mechanical ventilation: A prospective cohort study. J Crit Care [Internet]. 2015 Aug;30(4):862.e7-862.e13. Available from: https://linkinghub.elsevier.com/retrieve/pii/S088394411500146X

10. Elkins M, Dentice R. Inspiratory muscle training facilitates weaning from mechanical ventilation among patients in the intensive care unit: a systematic review. J Physiother [Internet]. 2015 Jul;61(3):125-34. Available from: https://linkinghub.elsevier.com/retrieve/pii/S1836955315000557 
11. Jeong BH, Ko MG, Nam J, Yoo H, Chung CR, Suh GY, et al. Differences in clinical outcomes according to weaning classifications in medical intensive care units. PLoS One. 2015;10(4):1-13.

12. Oliveira ABF de, Dias OM, Mello MM, Araújo S, Dragosavac D, Nucci A, et al. Fatores associados à maior mortalidade e tempo de internação prolongado em uma unidade de terapia intensiva de adultos. Rev Bras Ter Intensiva [Internet]. 2010 Sep;22(3):250-6. Available from: http://www.scielo.br/scielo.php? script=sci_arttext\&pid=S0103$507 \mathrm{X} 2010000300006 \& \operatorname{lng}=\mathrm{pt} \& \mathrm{nrm}=\mathrm{iso} \& \mathrm{tlng}=\mathrm{pt}$

13. Danaga AR, Gut AL, Antunes LC de O, Ferreira AL dos A, Yamaguti FA, Christovan JC, et al. Avaliação do desempenho diagnóstico e do valor de corte para o índice de respiração rápida e superficial na predição do insucesso da extubação. J Bras Pneumol [Internet]. 2009 Jun;35(6):541-7. Available from:

http://www.scielo.br/scielo.php?script=sci_arttext\&pid=S1806-

$37132009000600007 \& \operatorname{lng}=$ pt\&tlng $=$ pt

14. van Berkel A, van Lieshout J, Hellegering J, van der Hoeven JG, Pickkers P. Causes of death in intensive care patients with a low APACHE II score. Neth J Med [Internet]. 2012 Dec;70(10):455-9. Available from: http://www.ncbi.nlm.nih.gov/pubmed/23230015

15. Louriz M, Abidi K, Akkaoui M, Madani N, Chater K, Belayachi J, et al. Determinants and outcomes associated with decisions to deny or to delay intensive care unit admission in Morocco. Intensive Care Med [Internet]. 2012 May 8;38(5):830-7. Available from: http://link.springer.com/10.1007/s00134-012-2517-0

16. Souza S da S, Figueiredo LC de, Guedes CAV, Araújo S. Teste de permeabilidade de vias aéreas pré-extubação: comparação entre três métodos em ventilação espontânea. Rev Bras Ter Intensiva [Internet]. 2007 Sep;19(3):310-6. Available from: http://www.scielo.br/scielo.php?script=sci_arttext\&pid=S0103507X2007000300007\&lng=pt\&nrm=iso\&tlng=pt

17. Arcentales A, Caminal P, Diaz I, Benito S, Giraldo BF. Classification of patients undergoing weaning from mechanical ventilation using the coherence between heart rate variability and respiratory flow signal. Physiol Meas [Internet]. 2015 Jul 1;36(7):1439-52. Available from: http://stacks.iop.org/0967-

$3334 / 36 / \mathrm{i}=7 / \mathrm{a}=1439$ ?key=crossref.dbb8a16c3b5e2f17c76d2d9c5f7ebabe

18. Reis HFC dos, Almeida MLO, Silva MF da, Moreira JO, Rocha M de S. Association between the rapid shallow breathing index and extubation success in patients with traumatic brain injury. Rev Bras Ter Intensiva [Internet]. 2013;25(3):212-7. Available from: http://www.gnresearch.org/doi/10.5935/0103-507X.20130037

19. Menon N, Joffe AM, Deem S, Yanez ND, Grabinsky A, Dagal AH, et al. Occurrence and Complications of Tracheal Reintubation in Critically Ill Adults. Respir Care [Internet]. 2012 Oct 1;57(10):1555-63. Available from:

http://openurl.ingenta.com/content/xref?genre=article\&issn $=0020$ -

$1324 \&$ volume $=57 \&$ issue $=10 \&$ spage $=1555$

20. Savi A, Teixeira C, Silva JM, Borges LG, Pereira PA, Pinto KB, et al. Weaning predictors 
do not predict extubation failure in simple-to-wean patients. J Crit Care [Internet]. 2012 Apr;27(2):221.e1-221.e8. Available from:

https://linkinghub.elsevier.com/retrieve/pii/S0883944111003728

21. Jordan J, Rose L, Dainty KN, Noyes J, Blackwood B. Factors that impact on the use of mechanical ventilation weaning protocols in critically ill adults and children: A qualitative evidence-synthesis. Cochrane Database Syst Rev. 2015;2015(7).

22. Zhu B, Li Z, Jiang L, Du B, Jiang Q, Wang M, et al. Effect of a quality improvement program on weaning from mechanical ventilation: a cluster randomized trial. Intensive Care Med [Internet]. 2015 Oct 9;41(10):1781-90. Available from:

http://link.springer.com/10.1007/s00134-015-3958-z 